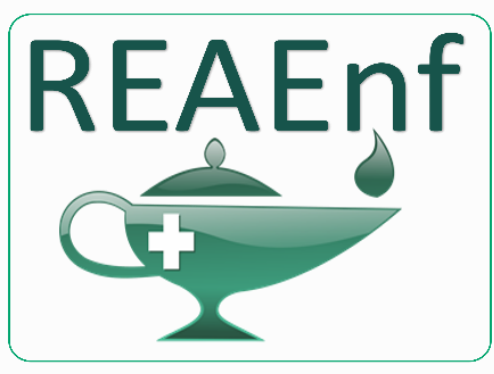

Revista Eletrônica Acervo Enfermagem

\section{RELATO DE CASO}

Recebido em: 1/2020

Aceito em: $1 / 2020$

Publicado em: $1 / 2020$

\title{
Diagnósticos, intervenções e resultados de enfermagem CIPE® a uma paciente com pielonefrite: relato de caso
}

\author{
CIPE® nursing diagnostics, interventions and results to a patient with pielonephritis: case \\ report
}

\author{
Diagnóstico de enfermería CIPEß, intervenciones y resultados a un paciente con \\ pielonefritis: reporte de caso
}

Brenner Kássio Ferreira de Oliveira ${ }^{1 *}$, Maxwell Arouca da Silva ${ }^{1}$, Alessandra da Silva Carvalho ${ }^{1}$, Raylesson Oliveira da Silva ${ }^{1}$, Amanda da Silva Melo ${ }^{1}$, Cliviane Farias Cordeiro ${ }^{1}$, Quéfren Guimarães Suzuki ${ }^{1}$, Priscilla Mendes Cordeiro'.

\begin{abstract}
Resumo: Este artigo buscou relatar a experiência da prática da sistematização da assistência de enfermagem, com base nas demandas terapêuticas de uma paciente com pielonefrite de acordo com a teoria das necessidades humanas básicas e a CIPE $®$. Trata-se de um relato de caso com abordagem descritiva de acadêmicos e docentes de um curso de graduação em enfermagem do interior do Amazonas. Diagnósticos, intervenções e resultados de enfermagem foram construídos a partir dos termos constantes no Modelo de Sete Eixos da CIPE® versão 2.0. Foram elaborados 9 Diagnósticos de Enfermagem (DE) diretamente relacionados ao processo patológico: Risco de Infecção aumentada por presença de acesso venoso periférico, eliminação urinária prejudicada, marcha prejudicada, ansiedade da separação aumentada, dor aguda moderada, falta de apetite, sono prejudicado, aceitação da condição de saúde prejudicada, higiene corporal prejudicada. $\mathrm{O}$ uso da CIPE $\circledast$ para construção das afirmativas diagnósticas, nos mostra a possibilidade de outros métodos para aplicação do processo de enfermagem, possuindo aplicação individual como coletiva, revelando-se um instrumento que pode contribuir com a organização e a qualidade do cuidado prestado, orientando e facilitando a tomada de decisões no ambiente hospitalar.
\end{abstract}

Palavras-chave: Pielonefrite, Enfermagem, CIPE.

\begin{abstract}
This article sought to report the experience of the practice of systematizing nursing care, based on the therapeutic demands of a patient with pyelonephritis according to the theory of basic human needs and the ICNP®. It is a case report with a descriptive approach by academics and professors of an undergraduate nursing course in the interior of Amazonas. Nursing diagnoses, interventions and results were constructed based on the terms contained in the CIPE® Seven Axis Model version 2.0. Nine Nursing Diagnoses (ED) were made directly related to the pathological process: increased risk of infection due to the presence of peripheral venous access, impaired urinary elimination, impaired gait, increased separation anxiety, moderate acute pain, lack of appetite, impaired sleep, acceptance impaired health condition, impaired body hygiene. The use of ICNP® for the construction of diagnostic statements, shows us the possibility of other methods for applying the nursing process, having individual and collective application, revealing itself as an instrument that can contribute to the organization and quality of the care provided, guiding and facilitating decision-making in the hospital environment.
\end{abstract}

Keywords: Pielonefrite, Nursing, ICNP.

${ }^{1}$ Universidade Federal do Amazonas (UFAM). Coari - Amazonas. *E-mail: brennerkassio@hotmail.com 
Resumen: Este artículo buscó informar la experiencia de la práctica de sistematizar la atención de enfermería, basada en las demandas terapéuticas de un paciente con pielonefritis de acuerdo con la teoría de las necesidades humanas básicas y el ICNP®. Es un informe de caso con un enfoque descriptivo por parte de académicos y profesores de un curso de pregrado en enfermería en el interior de Amazonas. Los diagnósticos, las intervenciones y los resultados de enfermería se construyeron con base en los términos contenidos en el CIPE® Seven Axis Model versión 2.0. Se realizaron nueve diagnósticos de enfermería (DE) directamente relacionados con el proceso patológico: mayor riesgo de infección debido a la presencia de acceso venoso periférico, eliminación urinaria alterada, marcha alterada, aumento de la ansiedad por separación, dolor agudo moderado, falta de apetito, falta de sueño, aceptación condición de salud deteriorada, higiene corporal deteriorada. El uso de ICNP $\AA$ para la construcción de declaraciones de diagnóstico, nos muestra la posibilidad de otros métodos para aplicar el proceso de enfermería, que tienen aplicación individual y colectiva, revelándose como un instrumento que puede contribuir a la organización y la calidad de la atención brindada, orientando y facilitando la toma de decisiones en el entorno hospitalario.

Palabras clave: Pielonefrito, Enfermería, ICNP.

\section{INTRODUÇÃO}

Infecção do trato urinário (ITU) é um termo relacionado a condições clínicas que podem ser classificadas como manifestações do trato inferior que envolve uretra e bexiga (uretrites e cistites) e infecções trato superior ou Pielonefrites que afeta a pelve e o parênquima renal. Essas áreas do sistema geniturinário podem sofrer com processos infecciosos ocasionados por bactérias do grupo gram negativas, sua maioria originadas do trato intestinal, onde se enquadra a bactéria Escherichia Coli (E. Coli), que representa 75 a $90 \%$ dos casos de ITU diagnósticos nos serviços de saúde (CASTELO CORRAL LBA, 2013; CALDERON-JAIMES E, 2013).

Mundialmente, a ITU é a segunda infecção mais detectada e tratada, perdendo somente para as respiratórias, representando um problema frequente de saúde. Esta acomete em sua maioria mulheres jovens com vida sexualmente ativa, mulheres grávidas e mulheres em pré - menopausa, representando $5 \%$ a $0,7 \%$ das notificações de infecções por ano (ARAUJO KL, QUEIROZ AC, 2012; COLOMB SALUD AS, 2014).

Dentre os processos patológicos, quando se menciona a infecção da pelve ou parênquima renal, denominados de pielonefrite, que em geral derivada do tráfego de bactérias do advindas da bexiga pelo ureter, tendo como principais manifestações clinicas a associação ou não com sintomas de cistite, além de febre, náuseas, êmese, calafrios e lombalgia (NICOLLE LE, 2009).

Considerando o tratamento, usualmente se faz a escolha da antibioticoterapia como terapêutica medicamentosa, elegendo-se os seguintes medicamentos como mais recomendados: sulfametoxazol trimetoprim, ciprofloxacina e ampicilina, são as terapias mais comumente recomendadas para Unidade de Terapia Intensiva (UTI), considerando-se a prescrição com análise do quadro clínico do paciente, história pregressa, exames de urina (MCGREGOR JE, et al., 2013), podendo ser realizado ou não o exame de sensibilidade ao antimicrobianos de espectro de ação apropriados ao tratamento das ITU, pois depende da disponibilidade do exame no local de habitação do paciente.

Cabe à equipe multidisciplinar atuar em suas respectivas áreas no diagnóstico e intervenções em saúde ao paciente com processos infecciosos e crônicos, na qual o bom acolhimento a este paciente, o direciona para um cuidado mais qualificado e humanizado, exige um conhecimento científico tão aprofundado quanto específico (COLOMBIANA DE SALUD AS, 2014).

A Enfermagem, como parte desta equipe, no decorrer dos anos, cada vez mais vem se firmando como ciência, arte e tecnologia nas dimensões individual e coletiva, com compromisso social firmado nas necessidades de saúde da população, na perspectiva de fundamentar o seu instrumento de estudo, considerado o cuidado em suas múltiplas faces de existência e aplicação (MOURA DJM, et al., 2014).

$O$ desenvolvimento de teorias como norteadoras para cuidado permite a enfermagem uma maior abrangência no contexto de saúde, onde se diferencia do modelo biomédico, observando não apenas as consequências das doenças, mas considerando a unicausalidade e a multicausalidade dentro do contexto 
dos determinantes de saúde. Nesses termos, foi criada a Teoria das Necessidades Humanas Básicas (NHB), com amplo espectro de aplicabilidade, visando o cuidado ao indivíduo, família ou comunidade, onde as necessidades podem ser iguais a todos, mas diferenciando no processo patológico apresentado por cada indivíduo (HORTA WA, 1970).

A enfermagem utiliza-se de seu método científico, chamado de processo de enfermagem (PE), para aplicar cuidados de forma sistematizada, além do uso de sistemas de classificações dos elementos de sua prática. Dentre esses sistemas, a Classificação Internacional para a Prática de Enfermagem (CIPE®), permite formular diagnósticos, resultados e intervenções, através de seus subconjuntos terminológicos, favorecendo o registro e uma melhoria na qualidade do atendimento, é uma classificação pouco conhecida e utilizada na prática clínica dos enfermeiros (BARRA DC, SASSO GT, 2012).

Assim, a relevância deste trabalho se mostra em visar o fortalecimento e melhoria do cuidado, através da associação entre o processo de enfermagem, taxonomias e teorias de enfermagem. Dessa forma, objetivase nesse estudo relatar a experiência da prática da sistematização da assistência de enfermagem, com base nas demandas terapêuticas de uma paciente com pielonefrite de acordo com a teoria das necessidades humanas básicas e a CIPE®, para assistência à saúde em uma unidade hospitalar.

\section{RELATO DE CASO}

O caso ao qual foi inspirado o relato corresponde ao de uma cliente, adulta jovem, com diagnóstico clínico de pielonefrite aguda, hospitalizado por um período de 7 dias. Para coleta de dados utilizou-se um histórico baseado no instrumento extraído do livro Anamnese e Exame Físico de Barros ALBL (2015) este, por sua vez, era composto por dados pessoais, queixas, história da doença atual, antecedentes pessoais fisiológicos, antecedentes pessoais patológicos, antecedentes familiares, hábitos de vida, situação socioeconômica e exame físico, norteado pela Teoria das Necessidades Humanas de Wanda de Aguiar Horta.

Diagnósticos, intervenções e resultados de enfermagem foram construídos a partir dos termos constantes no Modelo de Sete Eixos da CIPE® versão 2.0 (Foco, Julgamento, Cliente, Ação, Meios, Localização e Tempo) e as diretrizes do Conselho Internacional de Enfermeiros. Para formulação dos diagnósticos, se utiliza a inclusão obrigatória de um termo do eixo Foco e um termo do eixo Julgamento; e termos adicionais conforme a necessidade. Na elaboração das intervenções de enfermagem, utilizou-se obrigatoriamente um termo do eixo Ação e um termo do eixo Alvo (foco), considerado um termo de qualquer um dos eixos, exceto o eixo Julgamento. Utilizou-se a mesma estrutura dos diagnósticos para na elaboração dos resultados.

Diante dos vários problemas que podem ser identificados, foram elaborados 9 Diagnósticos de Enfermagem (DE) os quais podem ser identificados em pacientes com pielonefrite enquanto hospitalizados, diretamente relacionados ao processo patológico, os quais foram: Risco de Infecção aumentada por presença de acesso venoso periférico, eliminação urinária prejudicada, marcha prejudicada, ansiedade da separação aumentada, dor aguda moderada, falta de apetite, sono prejudicado, aceitação da condição de saúde prejudicada, higiene corporal prejudicada, estando estes classificados conforme a Teoria das Necessidades Básicas, na qual os DE formulados enquadram-se nas necessidades psicobiológicas e psicossociais. Os dados obtidos que norteiam esse relato estão apresentados em um quadro, onde constam os itens necessidades afetadas, diagnóstico de enfermagem, os resultados e intervenções realizadas podem ser visualizadas no Quadro 1. 
Quadro 1 - Diagnósticos, intervenções e resultados de enfermagem para portadora de pielonefrite utilizando a nomenclatura CIPE $\AA$, Coari - AM, 2019.

\begin{tabular}{|c|c|c|c|c|}
\hline $\begin{array}{c}\text { Necessidades humanas } \\
\text { básicas (nhb) }\end{array}$ & $\begin{array}{l}\text { Diagnóstico de } \\
\text { enfermagem }\end{array}$ & Achado clínico & Resultados esperados & Intervenções de enfermagem \\
\hline $\begin{array}{l}\text { Necessidade Psicobiológica - } \\
\text { Regulação Imunológica }\end{array}$ & $\begin{array}{l}\text { Risco de Infecção } \\
\text { aumentado por presença de } \\
\text { acesso venoso periférico. }\end{array}$ & Lesão na pele & Infecção Ausente & $\begin{array}{l}\text { Manter integridade da pele na via intravenosa } \\
\text { sempre. } \\
\text { Purificar a localização de dispositivo invasivo } \\
\text { com técnica asséptica. } \\
\text { Cuidados com local de dispositivo invasivo. } \\
\text { Colocar cobertura de ferida no local de } \\
\text { dispositivo invasivo. } \\
\text { Monitorar Sinais e Sintomas de Infecção. } \\
\text { Assegurar segurança do paciente durante } \\
\text { procedimentos invasivos. }\end{array}$ \\
\hline $\begin{array}{c}\text { Necessidade Psicobiológica - } \\
\text { Eliminação }\end{array}$ & $\begin{array}{l}\text { Eliminação urinária } \\
\text { prejudicada }\end{array}$ & Disúria & Micção eficaz & $\begin{array}{l}\text { Obter dados sobre condição urinária. } \\
\text { Orientar sobre irrigação da bexiga urinária. } \\
\text { Promover Eliminação urinária eficaz. } \\
\text { Executar educação em saúde sobre Sistema } \\
\text { Urinário. } \\
\text { Manter higiene íntima. } \\
\text { Orientar sobre o suprimento de água. } \\
\text { Fornecer suprimento de água adequado. }\end{array}$ \\
\hline $\begin{array}{l}\text { Necessidades Psicobiológica } \\
\text { - Atividade física, Mecânica } \\
\text { corporal, Motilidade e } \\
\text { Locomoção. }\end{array}$ & Marcha prejudicada & $\begin{array}{l}\text { Dor Lombar } \\
\text { Desconforto }\end{array}$ & Mobilidade melhorada & $\begin{array}{l}\text { Obter dados sobre capacidade para andar. } \\
\text { Orientar sobre técnica de deambulação. } \\
\text { Administrar medicação para dor. } \\
\text { Ajudar na deambulação. }\end{array}$ \\
\hline $\begin{array}{l}\text { Necessidade Psicobiológica - } \\
\text { Percepção dos órgãos dos } \\
\text { sentidos: olfativa, visual, } \\
\text { auditiva, tátil, gustativa, } \\
\text { dolorosa }\end{array}$ & Dor aguda moderada & $\begin{array}{l}\text { Dor lombar } \\
\text { Dor no flanco }\end{array}$ & Controle da dor. & $\begin{array}{l}\text { Explicar a causa da dor, se possível. } \\
\text { Estimular a verbalização da dor. } \\
\text { Registrar características da dor. } \\
\text { Avaliar eficácia da medicação, após sua } \\
\text { administração. }\end{array}$ \\
\hline
\end{tabular}

\footnotetext{
REAEnf/EJNC | Vol. 2 | e2900 | DOI: https://doi.org/10.25248/REAenf.e2900.2020 Página 4 de 8
} 
Revista Eletrônica Acervo Enfermagem / Electronic Journal Nursing Collection | ISSN 2674-7189

\begin{tabular}{|c|c|c|c|c|}
\hline $\begin{array}{c}\text { Necessidade Psicobiológica } \\
\text { - Nutrição }\end{array}$ & Falta de apetite aumentada & $\begin{array}{l}\text { Perda de peso } \\
\text { Inapetência }\end{array}$ & $\begin{array}{l}\text { Ingestão de alimentos } \\
\text { melhorada }\end{array}$ & $\begin{array}{l}\text { Obter dados sobre capacidade para alimentar- } \\
\text { se. } \\
\text { Auxiliar o paciente a se alimentar. } \\
\text { Solicitar avaliação do serviço de nutrição } \\
\text { Pesar o paciente diariamente. }\end{array}$ \\
\hline $\begin{array}{c}\text { Necessidade Psicobiológica } \\
\text { - Sono e repouso }\end{array}$ & Sono prejudicado & Insônia & Sono adequado & $\begin{array}{l}\text { Obter dados sobre o sono } \\
\text { Orientar sobre o sono } \\
\text { Encorajar repouso. } \\
\text { Obter dados sobre ambiente. } \\
\text { Técnicas de monitoramento do sono e } \\
\text { mudança no estilo de vida. }\end{array}$ \\
\hline $\begin{array}{c}\text { Necessidade Psicobiológica } \\
\text { - Cuidado corporal }\end{array}$ & Higiene corporal prejudicada & Higiene insatisfatória & $\begin{array}{l}\text { Higiene corporal } \\
\text { melhorada }\end{array}$ & $\begin{array}{l}\text { Obter dados sobre Padrão de higiene do } \\
\text { cliente. } \\
\text { Obter dados sobre o padrão de higiene bucal. } \\
\text { Auxiliar na higiene do corpo. } \\
\text { Auxiliar a arrumar-se. } \\
\text { Orientar família sobre padrão de higiene. } \\
\text { Orientar cliente sobre higiene na região íntima. }\end{array}$ \\
\hline $\begin{array}{l}\text { Necessidade Psicossociais - } \\
\text { Liberdade e participação }\end{array}$ & $\begin{array}{l}\text { Aceitação da condição de } \\
\text { saúde prejudicada. }\end{array}$ & Irritação & $\begin{array}{l}\text { Aceitação da condição de } \\
\text { saúde melhorada }\end{array}$ & $\begin{array}{l}\text { Avaliar resposta ao tratamento. } \\
\text { Facilitar acesso ao tratamento. } \\
\text { Explicar todas as dúvidas do paciente. } \\
\text { Facilitar Capacidade da Família para } \\
\text { Participar no Plano de Cuidado }\end{array}$ \\
\hline $\begin{array}{l}\text { Necessidade Psicossocial - } \\
\text { Segurança emocional }\end{array}$ & $\begin{array}{c}\text { Ansiedade da Separação } \\
\text { aumentada. }\end{array}$ & $\begin{array}{l}\text { Desamparo } \\
\text { Sofrimento }\end{array}$ & Ansiedade reduzida & $\begin{array}{l}\text { Obter dados sobre Ansiedade do indivíduo. } \\
\text { Gerenciar ansiedade. } \\
\text { Proporcionar bem-estar. }\end{array}$ \\
\hline
\end{tabular}

Fonte: Oliveira BKF, et al., 2019.

REAEnf/EJNC | Vol. 2 | e2900 | DOI: https://doi.org/10.25248/REAenf.e2900.2020 Página 5 de 8 


\section{DISCUSSÃO}

Durante o processo de internação da paciente, um procedimento rotineiramente utilizado e observado no Hospital é o Acesso Venoso Periférico (AVP). Artifício este que se constitui na inserção de um AVP no vaso sanguíneo periférico, todavia, o uso deste pode ocasionar complicações locais ou sistêmicas, dentre ela o risco de infecção pode ser originado. Talvez este seja uma das mais comuns infecções que ocorre no ambiente hospitalar, mas, sendo "tão rotineira" esta deve ser evitada por seu grande número de casos (JOHANN DA, et al., 2016). Ações preventivas podem ser adotadas para que não venham a acontecer, dentre elas seria manter a integridade da pele na via intravenosa do sujeito o mais integra possível, aplicando técnicas sépticas e assépticas na região, além da averiguação constante do local da punção, ato que passa despercebido pela equipe.

A pielonefrite causa complicações do quadro clínico do sujeito além de distúrbios fisiológicos como a disúria, originando o diagnóstico de eliminação urinária prejudicada, comprometendo a qualidade de vida do indivíduo. Por isso, quanto antes diagnosticada, mais fácil e rápido será obtido a homeostasia corporal do ser acometido (BARROS SRAF, 2013). A disúria poderá causar um desconforto durante a micção, com isso o paciente evitará $o$ ato em si, em consequência de tal irritação, causando uma micção ineficaz. Com base nisso uma das intervenções utilizadas foi obter, catalogar e analisar dados sobre a condição urinária, e tentar promover uma eliminação vesical mais eficaz, orientar a paciente sobre a boa higiene íntima e hidratação eficaz.

A mobilidade física prejudicada pode surgir de forma imediata ou de forma tardia, o que vai determinar isso será a duração e a extensão de onde se encontra o agente estressor de tal fator. Essa limitação física pode ser vista como estopim desencadeador para outras complicações em diversos sistemas do corpo, mas o principal sistema acometido é o musculoesquelético (FRANÇA MJDM, et al., 2013). No caso clínico, a paciente sentia desconforto ao realizar a deambulação, praticando-a e/ou tornando-a de forma errônea por conta disso, que em muitos casos pode levar a não se sentir mais disposta por conta da dor, derivadas dos rins, órgãos que se encontram no flanco direito e esquerdo, quadrante que movimenta bastante durante a deambulação ou marcha. O profissional de enfermagem dever tomar medidas cabíveis dentre os quais a administração de medicamentos prescritos pela equipe médica para o alivio da dor seria uma atitude de forma primária e imediata, outro postulado seria coletar dados sobre a capacidade de deambular e orientar técnicas de deambulação mostraria tão eficaz quanto a anterior, e se necessário ajudar na marcha da paciente.

No âmbito hospitalar e/ou clínica, o paciente bem como os familiares que o acompanham em muitos dos casos sente-se desamparados, a multiplicidade dos problemas e as alterações fisiológicas, desafia os profissionais da saúde a uma abordagem mais holística (FARIA JMS, PONTIFICE-SOUSA P, GOMES MJP, 2018). A paciente do estudo sentia-se uma sensação de desamparo e solidão, acarretando desse modo o aumento do sofrimento psíquico. Por isso cabe ao profissional tentar eliminar ou reduzir esse sentimento, sendo uma das principais intervenções de enfermagem observar e catalogar dados sobre a ansiedade do indivíduo, gerenciar e tentar amenizar o surgimento da mesma proporcionando uma sensação de bem estar.

O desconforto da doença, o ambiente não familiar ou mais aconchegante, pode ocasionar a perda de apetite. A adequação da ingestão de alimentos, obtenção de dados sobre a capacidade alimentar do indivíduo, ou até mesmo auxiliar o paciente a se alimentar, solicitando avaliação do serviço de nutrição para verificação de uma dieta que facilite a aceitabilidade do cliente, além do controle do peso e índice de massa corporal, são intervenções que podem ser implementadas pela equipe de enfermagem.

O sono é extremamente importante para a recuperação corporal e equilíbrio homeostático principalmente em uma situação de doença, é importante também para prevenir o surgimento de novas enfermidades, pois uma qualidade de sono bom ajuda na regulação e melhora o sistema imunológico e humoral (MONTEIRO NT, CEOLIM MF, 2014). A paciente do estudo possuía um padrão de sono prejudicado caracterizado por quadros de insônia, com base nisso, proporcionar um sono adequado, a fim de sanar esses episódios de insônia, forma prioridade dentre as intervenções, assim obtiveram-se dados sobre o sono, orientou-se a importância do sono, encorajando o repouso, verificando uma adaptação do ambiente aos critérios da cliente, aplicando técnicas de monitoramento do sono e o incentivo a mudança no estilo de vida do paciente.

REAEnf/EJNC | Vol. 2 | e2900 | DOI: https://doi.org/10.25248/REAenf.e2900.2020 Página 6 de 8 
A higiene de modo geral tem sido assunto bastante discutido na enfermagem, alguns determinantes que influenciam intrinsicamente nesse processo, dentre eles hábitos e costumes culturais ganham maior destaque e enfoque (CARRASCAL GC, RAMIREZ JDM, 2015). A higiene bem como sua atividade e desempenho também variam conforme o quadro clínico do paciente, podendo ser um determinante de estrema pertinência. No presente caso, a paciente se encaixa neste último eixo possuindo uma higiene insatisfatória. Com isso cabe ao profissional reverter este presente quadro proporcionando uma higiene corporal melhorada aplicando algumas intervenções, como verificação do padrão de higiene da cliente corporal e oral, se necessário auxiliar em sua higienização e ao vestir-se.

Evidencia-se que o diagnóstico de enfermagem é um processo cognitivo dependente do agrupamento da coleta, da qualidade da análise de dados, geração e suposição e análise de hipóteses, contribui com a comunicação, norteia os resultados esperados e as intervenções para alcançá-los (BARROS AL, et al., 2015)

A Teoria de Horta WA (1970) e a CIPE $®$ nesse contexto, auxilia a equipe de enfermagem a buscar cuidar do cliente na ênfase de suas necessidades básicas. Atualmente, no processo de enfermagem, essa teoria é referência de muitas instituições hospitalares que utilizam seus conceitos em uma elevação do nível de consciência e de ação da enfermagem e da equipe multiprofissional. Pires SMB, et al. (2011) frisa que entender o ser humano considerando o corpo, mente e espírito, permite compreender em um sentido mais amplo o significado de saúde e cuidado, pois quando o corpo ou a mente sofre, a pessoa é afetada em sua totalidade. Há muito mais a ser considerado do que apenas as partes que a incomodam, no processo de cuidar de um ser também devem considerados os aspectos sociais, emocionais, para que o seu atendimento se torne um acolhimento individualizado e humanizado.

\section{CONSIDERAÇÕES FINAIS}

A utilização do processo de enfermagem vinculado a Sistematização da Assistência de Enfermagem (SAE) proporciona a equipe de enfermagem autonomia em seu trabalho, permitindo a adesão de práticas cientificas durante a assistência, sendo uma forma de documentação da atuação do profissional, valorizando a enfermagem frente à sociedade. Desta forma, através dos diagnósticos, resultados e intervenções mencionadas a paciente com pielonefrite aguda, obteve uma melhor adesão ao tratamento, verbalizou seus sentimentos através da comunicação assertiva, fortalecendo o vínculo com profissional cuidador, havendo diminuição do processo de ansiedade, demonstrando interesse em adotar condutas que antes não faziam parte de sua rotina. Além disso, o uso da CIPE® para construção das afirmativas diagnósticas, nos mostra a possibilidade de outros métodos para aplicação do processo de enfermagem, possuindo aplicação individual como coletiva, revelando-se um instrumento que pode contribuir com a organização e a qualidade do cuidado prestado, orientando e facilitando a tomada de decisões no ambiente hospitalar. Por fim, espera-se que este trabalho, possa estimular a utilização de taxonomias durante o uso do processo de enfermagem, subsidiar pesquisas na área e aumentar relatos de casos de experiências acadêmicas, e que estas possam ser um meio de obter melhorias da qualidade de vida de pacientes, além da satisfação dos profissionais.

\section{REFERÊNCIAS}

1. ARAUJO KL, QUEIROZ AC. Análise do perfil dos agentes causadores de infecção do trato urinário e dos pacientes portadores, atendidos no Hospital e Maternidade Metropolitana - SP. J Health Sci Inst. 2012.

2. BARRA DC, SASSO GT. The nursing process according to the international classification for nuring practice: an integrative review. Texto Contexto Enferm. 2012; 21(2):440-7.

3. BARROS AL, et al. Processo de enfermagem: guia para a prática. São Paulo: COREN-SP; 2015.

4. BARROS ALBL. Anamnese e exame físico: avaliação diagnóstica de enfermagem no adulto. 3ํo ed. Porto Alegre: Artmed, 2015.

5. BARROS SRAF. Infecção urinária na gestação e sua correlação com a dor lombar versus intervenções de enfermagem. Rev Dor. São Paulo, 2013 abr-jun;14(2):88-93.

REAEnf/EJNC | Vol. 2 | e2900 | DOI: https://doi.org/10.25248/REAenf.e2900.2020 Página 7 de 8 
6. CALDERON-JAIMES E. Diagnóstico y tratamiento de las infecciones en vías urinarias: un enfoque multidisciplinario para casos no complicados. Bol Med Hosp Inafnt Mex, 3-10, 2013.

7. CARRASCAL GC, RAMIREZ JDM. Higiene: Cuidado básico que promueve la comodidade em pacientes críticos. Enfermería Global, 14(40), 340-350, 2015.

8. CASTELO CORRAL LBA. Recomendaciones prácticas para el diagnóstico y tratamiento de la infección urinaria en el adulto. Galicia clínica/sociedad de galega de medicina interna, 115-125, 2013.

9. COLOMBIANA DE SALUD SA. Guía de infecciones de vías urinarias en adultos. Bogotá: Colombiana de salud, 2014.

10. COMITÉ INTERNACIONAL DE ENFERMEIROS. CIPE, Versão 2.0: Classificação Internacional para a Prática de Enfermagem. São Paulo: Algol Editora; 2011.

11. FARIA JMS, PONTIFICE-SOUSA P, GOMES MJP. La comodidade del paciente en cuidados intensivos - una revisión integradora. Enfermería Global 17(50), 477-514, 2018.

12. FRANÇA MJDM, et al. Diagnósticos de enfermagem de pacientes com necessidade de locomoção afetada internados em uma unidade hospitalar. Rev. Eletr. Enf. 2013 out/dez;15(4):878-85.

13. HORTA WA. Processo de enfermagem. São Paulo: EPU, 104p, 1970.

14. JOHANN DA, et al. Fatores de risco para complicações no cateter venoso periférico em adultos: análise secundária de ensaio clínico randomizado. Rev. Latino-Am. Enfermagem 2016; 24 : e2833.

15. MCGREGOR JC, et al. Sex- and age-specific trends in antibiotic resistance patterns of Escherichia coli urinary isolates from outpatients. BMC Fam. Pract. v.14, n.25, 2013.

16. MONTEIRO NT, CEOLIM MF. Qualidade do Sono de Idosos no domicílio e na hospitalização. Texto Contexto Enferm, Florianópolis, 2014. Abr-Jun; 23(2): 356-64.

17. MOURA DJM, et al. Sistematização da assistência de enfermagem fundamentada na CIPE® e na teoria da adaptação em hipertensos. Rev. Eletr. Enf. out/dez;16(4):710-9, 2014.

18. NICOLLE LE. Uncomplicated urinary tract infection in adults including uncomplicated pyelonephritis. Urol Clin North Am.; 35(1): 1 - 12, 2009.

19. PIRES SMB, MÉIER MJ, DANSKI MTR. Fragmentos da trajetória pessoal e profissional de Wanda Horta: contribuições para a área da enfermagem. Revista Eletrônica. 2, n. 1, 2011. 